KAIST-TH 98/18

hep-ph/9809286

\title{
Small Instanton Contribution to the Axion Potential in Supersymmetric Models
}

\author{
Kiwoon Choi* and Hyungdo Kim ${ }^{\dagger}$ \\ Department of Physics, Korea Advanced Institute of Science and Technology \\ Taejon 305-701, Korea
}

\begin{abstract}
Small size QCD instantons may spoil the axion solution to the strong CP problem if QCD is not asymptotically free at high energy scales. We examine this issue in supersymmetric models using a manifestly supersymmetric scheme to compute the axion potential induced by small size instantons. Applying this scheme for a class of illustrative models, it is found that the resulting high energy axion potential is highly model-dependent, but suppressed by more powers of the soft supersymmetry breaking parameters and/or of the other small mass scales than what is expected based on a naive instanton graph analysis. Our analysis suggests that the axion solution is stable against the small QCD instanton effects in a wide class of supersymmetric models even when QCD is not asymtotically free at high energy scales.
\end{abstract}

PACS Number(s):

*E-mail: kchoi@higgs.kaist.ac.kr

†E-mail: hdkim@supy.kaist.ac.kr 


\section{INTRODUCTION}

One of the most attractive solutions to the strong $\mathrm{CP}$ problem [1] is to introduce a spontaneously broken $U(1)_{P Q}$ symmetry whose explicit breaking is entirely given by the QCD anomaly [2]. Once such a global $U(1)_{P Q}$ is assumed, it is usually taken for granted that $\theta_{Q C D}$ is dynamically relaxed down to a sufficiently small value by the vacuum expectation value (VEV) of the associated pseudo-Goldstone boson, the axion. However there are some class of models that this may not be true. If QCD is not asymptotically free at energy scales above the weak scale, small size QCD instantons may induce a significant high energy axion potential $V_{\mathrm{HE}}$ whose minimum does not coincide with the minimum of the conventional low energy axion potential $V_{\mathrm{LE}}$. If $V_{\mathrm{HE}} \gtrsim 10^{-9} V_{\mathrm{LE}}$, the dynamical relaxation mechanism of $\theta_{Q C D}$ would be spoiled and then one looses the original motivation to introduce $U(1)_{P Q}$.

Perhaps the most interesting class of models in which the effects of small size instantons can be sizable are supersymmetric models. Some supersymmetric Grand Unified Theories (GUTs) like $E_{6}$ and also many supersymmetric models derived from superstring theories contain extra colored multiplets which would dramatically change the QCD $\beta$ function at energy scales above the weak scale, even making it positive in some cases. In fact, small size instanton contribution to the axion potential in models with extra colored particles has been studied before [3, [4] based on a simple dimensional analysis for instanton graphs. However, when applied for supersymmetric models, this scheme can overlook a possible cancellation between different instanton graphs which is due to supersymmetry (SUSY), thereby yielding a highly overestimated axion potential. To avoid this difficulty, in this paper we introduce a manifestly supersymmetric scheme to compute the axion potential and apply it for a class of illustrative models. Although an explicit analysis is made only for a limited class of models and also the resulting axion potential is highly model-dependent, our analysis suggests that the effects of small size instantons in supersymmetric models are much more suppressed than what is expected based on a naive instanton graph analysis. This implies that the axion solution to the strong CP problem is stable against the effects of small instantons in a wide class of SUSY models even when QCD is not asymtotically free at high energy scales.

To proceed, let us discuss in more detail how the dynamical relaxation of $\theta_{Q C D}$ can be spoiled by the high energy axion potential induced by small size QCD instantons. At the axion scale $f_{a}$ where $U(1)_{P Q}$ is spontaneously broken, the axion effective lagrangian is given by

$$
\mathcal{L}_{\text {axion }}=\frac{1}{2}\left(\partial_{\mu} a\right)^{2}+\frac{1}{f_{a}} \partial_{\mu} a J^{\mu}+\frac{1}{32 \pi^{2}} \frac{a}{f_{a}} F \tilde{F},
$$

where $J^{\mu}$ denotes a generic current in the model, and $F$ and $\tilde{F}$ are the gluon field strength and its dual, respectively. Integrating out the degrees of freedom at energy scales above the weak scale $M_{W}$, the nonderivative axion coupling to the QCD anomaly, i.e. $a F \tilde{F}$, would induce a high energy axion potential $V_{\mathrm{HE}}$ through the QCD instantons with size $\rho \ll M_{W}^{-1}$ :

$$
V_{\mathrm{HE}}=-V_{0} \cos \left(a / f_{a}-\gamma\right) \text {. }
$$

Both the size of this axion potential $\left(V_{0}\right)$, and the location of the minimum $(\gamma)$, depend upon the details of the physics at high energy scales around $\rho^{-1}$. Unlike $V_{\mathrm{HE}}$, the low energy axion potential induced by the dynamics below the weak scale can be unambiguously computed. 
Applying the chiral perturbation theory for the low energy QCD instanton amplitudes, one finds [1]

$$
V_{\mathrm{LE}}=-f_{\pi}^{2} m_{\pi}^{2} \sqrt{m_{u}^{2}+m_{d}^{2}+2 m_{u} m_{d} \cos \left(a / f_{a}\right)} /\left(m_{u}+m_{d}\right),
$$

where $m_{u}$ and $m_{d}$ denote the current masses of the up and down quarks, $f_{\pi}=94 \mathrm{MeV}$ and $m_{\pi}$ are the pion decay constant and mass, respectively. Here the axion VEV $\left(\left\langle a / f_{a}\right\rangle\right)$ is defined to correspond to the QCD vacuum angle which is required to be less than $10^{-9}$ from the non-observation of the neutron electric dipole moment:

$$
\theta_{Q C D}=\left\langle a / f_{a}\right\rangle \lesssim 10^{-9} .
$$

The axion VEV is then determined by the total axion potential $V_{\text {axion }}=V_{\mathrm{LE}}+V_{\mathrm{HE}}$. Obviously, in order for the axion VEV to satisfy the above phenomenological bound, the high energy axion potential has to be suppressed as $V_{0} / f_{\pi}^{2} m_{\pi}^{2} \lesssim 10^{-9} \gamma^{-1}$. If one wishes to achieve CP-conserving QCD independently of the details of $\mathrm{CP}$ violation at high energy scales, i.e. independently of $\gamma$, one would need

$$
\delta \equiv \frac{V_{\mathrm{HE}}}{10^{-9} f_{\pi}^{2} m_{\pi}^{2}} \lesssim 1 .
$$

In fact, there is a negligibly small extra low energy potential [0]

$$
\delta V_{\mathrm{LE}} \simeq 10^{-14} f_{\pi}^{2} m_{\pi}^{2} \sin \delta_{K M} \sin \left(a / f_{a}\right)
$$

due to the low energy dynamics involving the $\mathrm{CP}$ violation by the Kobayashi-Maskawa phase $\delta_{K M}$. This would shift the axion VEV also, but the resulting shift $\left\langle\delta a / f_{a}\right\rangle \simeq 10^{-14} \sin \delta_{K M}$ is well within the experimental bound.

In the next section, we describe how the instanton-induced $V_{\mathrm{HE}}$ can be computed in generic supersymmetric models in a manifestly supersymmetric way. In sect. 3, we apply this method for a class of illustrative SUSY models including extra quark multiplets and make a numerical estimate of the largest possible $V_{\mathrm{HE}}$. Based on this analysis, we conclude in sect. 4 that the axion solution to the strong CP problem is stable against the effects of small instantons in a wide class of SUSY models even when QCD is not asymtotically free at high energy scales.

\section{SMALL INSTANTON INDUCED AXION POTENTIAL IN SUSY MODELS}

In this section, we discuss how the high energy axion potential induced by small size QCD instantons can be computed in a manifestly supersymmetric way. Generic supersymmetric models contain a SUSY breaking sector which would provide a dynamical seed for SUSY breaking. The resulting SUSY breaking is transmitted to the observable sector through some messenger interactions characterized by the messenger scale $M_{m}$. Integrating out the fields in SUSY breaking sector and also in the messenger sector, one is left with an effective lagrangian of the observable sector fields in which SUSY appears to be softly broken. This effective lagrangian describes the observable sector dynamics at energy scales below $M_{m}$. The size of the messenger scale $M_{m}$ depends upon the character of messenger interactions. 
In gravity-mediated models [6], $M_{m}$ is given by the Planck scale $M_{P}$, while in gauge-mediated models [7] $M_{m}$ is somewhat model-dependent but typically far below $M_{P}$. At any rate, soft SUSY breaking in the observable sector is operative only at energy scales below $M_{m}$. As a result, QCD instantons with size $\rho \lesssim M_{m}^{-1}$ do not contribute to the axion potential due to SUSY fermion zero modes, e.g. gluino zero modes. To be definite, here we concentrate on gravity-mediated models, however our study can be easily applied for gauge-mediated models also.

Our starting point is the effective lagrangian of observable sector fields at energy scales around $\rho^{-1}$ where $\rho$ denotes the size of the small QCD instantons:

$$
\mathcal{L}=\int d^{2} \theta d^{2} \bar{\theta} \eta \Phi_{i}^{\dagger} \Phi_{i}+\frac{1}{32 \pi^{2}}\left[\int d^{2} \theta Y W^{\alpha} W_{\alpha}+\text { h.c. }\right]+\left[\int d^{2} \theta W\left(\Phi_{i}, Z\right)+\text { h.c. }\right] .
$$

Here $\Phi_{i}$ denote generic chiral superfields in the model, $W_{\alpha}$ are the spinorial chiral superfields for the gauge multiplets, $\eta$ is a real spurion superfield whose auxiliary $D$-component corresponds to the soft scalar mass, and finally $Y$ and $Z$ stand for the chiral spurions whose auxiliary $F$-components correspond to the gaugino mass and the soft $A$ (or $B$ ) coefficients, respectively. Explicitly, we have

$$
\begin{aligned}
\eta & =1+m_{0}^{2} \theta^{2} \bar{\theta}^{2}, \\
Y & =\frac{8 \pi^{2}}{g^{2}}+i \theta_{Q C D}+16 \pi^{2} m_{1 / 2} \theta^{2}, \\
Z & =\left\{Z_{\lambda}=\left(1+A \theta^{2}\right) \lambda, \quad Z_{\mu}=\left(1+B \theta^{2}\right) \mu\right\},
\end{aligned}
$$

where $\lambda$ and $\mu$ denote the trilinear Yukawa couplings and the bilinear $\mu$-parameter in the superpotential. It is assumed that all soft breaking terms are described by the universal soft scalar mass $m_{0}$, the universal gaugino mass $m_{1 / 2}$, and the universal trilinear $A$ and bilinear $B$ coefficients, which are presumed to have the weak scale values. The size of the small instanton-induced axion potential is not sensitive to the detailed form of the soft parameters and thus its order of magnitude can be estimated in the scheme assuming a universal form of soft parameters.

Once the effective lagrangian at energy scales around $\rho^{-1}$ is given as (6), the low energy interactions induced by small QCD instantons can be summarized by [8]

$$
\begin{aligned}
\mathcal{L}_{\mathrm{ins}} & =\int d^{2} \theta d^{2} \bar{\theta}\left[e^{-n_{1} Y} K_{\mathrm{eff}}\left(\Phi_{i}, \Phi_{i}^{*} ; D_{\alpha}, \bar{D}_{\alpha} ; Z, \bar{Z}^{*} ; \eta\right)+h . c .\right] \\
& +\left[\int d^{2} \theta e^{-n_{2} Y} W_{\mathrm{eff}}\left(\Phi_{i}, Z\right)+h . c .\right]
\end{aligned}
$$

where $n_{1}$ and $n_{2}$ are positive integers corresponding to the instanton winding number and $D_{\alpha}$ denotes the supercovariant derivative acting on either superfields or spurions. In order to generate an axion potential, all external fields with vanishing VEVs, e.g. external quark superfields, in the instanton amplitudes have to be integrated out to induce a term including only the superfields with nonvanishing VEVs. Then the axion potential can be derived from the terms in $W_{\text {eff }}$ or $K_{\text {eff }}$ by replacing all the superfields by their VEVs, the spurion $Y$ by $Y+i a / f_{a}$, and finally integrating out the Grassmann coordinates $\theta$ and $\bar{\theta}$. Note that the supercovariant derivatives are not allowed to appear in $W_{\text {eff }}$ since $\bar{D} \Phi=\bar{D} Z=0$ and also $D^{2} \Phi\left(D^{2} Z\right)$ is not a chiral superfield (spurion). 
Let $m_{\text {soft }}$ collectively denote the soft mass parameters, i.e.

$$
\left\{m_{\text {soft }}\right\}=\left\{m_{0}, m_{1 / 2}, A, B\right\} .
$$

Usually any nonzero VEV of the auxiliary $F$-component of the observable sector field $\Phi_{i}$ is due to the presence of soft SUSY breaking terms and thus it is suppressed by some powers (typically a single power) of $m_{\text {soft }}$, e.g.

$$
\left\langle\Phi_{i}\right\rangle \approx\left(1+m_{\mathrm{soft}} \theta^{2}\right)\left\langle\phi_{i}\right\rangle
$$

where $\phi_{i}$ is the scalar component of the superfield $\Phi_{i}$. One simple consequence of this observation is that the axion potential arising from $K_{\text {eff }}$ is suppressed at least by two powers of $\rho m_{\text {soft }}$ since it is obtained after the Grassmann integration over $d^{2} \theta d^{2} \bar{\theta}$, while the axion potential from $W_{\text {eff }}$ is suppressed at least by a single power of $\rho m_{\text {soft }}$.

The instanton-induced effective Kähler potential $K_{\text {eff }}$ and the effective superpotential $W_{\text {eff }}$ can be systematically computed by expanding them in powers of the small dimensionless quantities: $\rho \Phi_{i}, \rho D_{\alpha} D^{\alpha}, Z_{\lambda}$, and $\rho Z_{\mu}$. In fact, such an expansion is severely constrained by the selection rules dictated by the internal symmetries of the tree level superpotential $W\left(\Phi_{i}, Z\right)$ in (6) under which both the superfields and spurions transform. Another useful requirement is that it should have a sensible limiting behavior when some of the couplings are turned off. As we will see, together with the holomorphy, these requirements forbid $W_{\text {eff }}$ to include a term which would contribute to the axion potential by replacing the superfields by their VEVs. As a result, the leading contribution to the axion potential comes from the effective Kähler potential $K_{\mathrm{eff}}$ and thus is suppressed at least by two powers of $\rho m_{\mathrm{soft}}$.

Once the superfields in $K_{\text {eff }}$ are replaced by their VEVs and also the Grassmann integration is performed, the expansion in powers of $\rho \Phi_{i}, \rho D^{\alpha} D_{\alpha}, \rho Z_{\mu}$, and $Z_{\lambda}$ appear to be an expansion in $\left\langle\rho \phi_{i}\right\rangle, \rho m_{\text {soft }}, \rho \mu$ and also the Yukawa couplings in the model. Generically the field VEVs are calculable in terms of $m_{\text {soft }}$ and/or the other mass parameters, e.g. $\mu$ and $M_{P}$, in the model. One can then easily identify the dominant term by counting the powers of $\rho m_{\text {soft }}$ and $\rho \mu$ (and also of the Yukawa couplings if necessary) in the resulting axion potential. Although the selection rules determine how the high energy axion potential depends upon the various parameters and the VEVs, they do not fix the additional suppression factor arising from the loops in the instanton graph. The axion potential induced by small instantons is essentially given by closing the fermionic zero modes in the instanton-induced multiple fermion operator in the dilute gas approximation. Once the dominant term in the expansion of $K_{\text {eff }}$ were identified, the corresponding instanton graph can be identified also. This allows us to estimate the loop suppression factor by counting the number of loops in the graph, thereby completing the estimate of the size of the high energy axion potential.

The above procedure computing the instanton-induced axion potential is based on a manifestly supersymmetric formulation and thus automatically takes into account the possible cancellation dictated by SUSY. Anticipating SUSY cancellation between different instanton graphs would be highly nontrivial in a naive diagrammatic analysis. In fact, in many cases one can draw an instanton graph which is consistent with the selection rules and the sensible limiting behavior, but with a less powers of $\rho m_{\text {soft }}$ than those required by SUSY. This implies that such graph is cancelled by other instanton graphs which are related to the original graph by SUSY. Our method automatically avoids such misleading graphs since it includes only the instanton graphs allowed by both SUSY and the selection rules. Following 
the recipe described above, in the next section we will compute the small instanton-induced axion potential in a class of illustrative SUSY models.

\section{COMPUTATION AND NUMERICAL ANALYSIS}

There are many possible supersymmetric extensions of the Standard Model, starting from the minimal supersymmetric standard model (MSSM) to other models including additional matter multiplets. Here we are mainly interested in models with additional colored multiplets which would change the QCD $\beta$ function at high energy scales, thereby leading to a sizable high energy axion potential generated by small QCD instantons. To examine this possibility, in this section we consider a model containing $N_{h}$-flavors of isosinglet quark and antiquark multiplets $\left(h+h^{c}\right)$ and also a gauge-singlet superfield $(S)$ in addition to the 3 generations of the MSSM quark multiplets (isodoublet quarks $Q$ and isosinglet antiquarks $\left.U^{c}+D^{c}\right)$. This model is a rather simple generalization of the MSSM, but still shows many of the essential features of the axion potential induced by small instantons in generic SUSY models.

The tree level superpotential of our model includes

$$
W=Z_{u} Q H_{u} U^{c}+Z_{d} Q H_{d} D^{c}+Z_{h} \frac{S^{k+1}}{M_{P}{ }^{k}} h h^{c}+Z_{1} \frac{S^{n+1}}{M_{P}{ }^{n}} H_{u} H_{d}+Z_{2} \frac{S^{l+3}}{M_{P}{ }^{l}},
$$

where $l, k, n$ are non-negative integers, and $H_{u}$ and $H_{d}$ denote the isodoublet Higgs superfields. Terms involving lepton superfields are irrelevant for the analysis of the axion potential and thus are ignored. Here $Z_{u, d}$ are $3 \times 3$ matrices, $Z_{h}$ is $N_{h} \times N_{h}$ matrix, and all couplings are spurions which are determined by the Yukawa couplings and the universal $A$-coefficient:

$$
\begin{aligned}
& Z_{u}=\lambda_{u}\left(1+A \theta^{2}\right), \quad Z_{d}=\lambda_{d}\left(1+A \theta^{2}\right) \\
& Z_{1}=\lambda_{1}\left(1+A \theta^{2}\right), \quad Z_{2}=\lambda_{2}\left(1+A \theta^{2}\right), \\
& Z_{h}=\lambda_{h}\left(1+A \theta^{2}\right) .
\end{aligned}
$$

A characteristic feature of the models considered here is that all mass scales are determined in terms of the two basic mass scales $m_{\text {soft }}$ and $M_{P}$ and also the three non-negative integers $(l, k, n)$. (See Eqs. (14) and (15).) It is straightforward to repeat our following analysis for other type of models including a mass scale which is not related with $m_{\text {soft }}$ and $M_{P}$.

To proceed, let us first consider the ground state configuration of the model. We assume that $S$ has a sizable renormalizable Yukawa coupling, for instance the Yukawa coupling $\lambda_{h}$ with $h+h^{c}$ in the case of $k=0$, or a renormalizable Yukawa coupling with lepton-like superfields in other cases with $k>0$. This would make the running soft mass of $S$ to become negative at energy scales around $\langle S\rangle$. Then the effective potential of $S$ is schematically given by

$$
V \approx-m_{\mathrm{soft}}^{2} S^{2}+\frac{S^{2(l+2)}}{M_{P}^{2 l}}
$$

where we have omitted the coefficients of order unity. The resulting VEV of $S$ is 


$$
\langle S\rangle \approx M_{P}\left(\frac{m_{\mathrm{soft}}}{M_{P}}\right)^{\frac{1}{l+1}},
$$

yielding the supersymmetric masses $\left(\mu_{H}\right.$ and $\left.\mu_{h}\right)$ of $H_{u}+H_{d}$ and $h+h^{c}$ as follows:

$$
\begin{aligned}
& \mu_{H}=\lambda_{1} \frac{\langle S\rangle^{n+1}}{M_{P}{ }^{n}} \approx m_{\mathrm{soft}}\left(\frac{m_{\mathrm{soft}}}{M_{P}}\right)^{\frac{n-l}{l+1}} \\
& \mu_{h}=\lambda_{h} \frac{\langle S\rangle^{k+1}}{M_{P}{ }^{k}} \approx m_{\mathrm{soft}}\left(\frac{m_{\mathrm{soft}}}{M_{P}}\right)^{\frac{k-l}{l+1}}
\end{aligned}
$$

where we have assumed that $\lambda_{1}, \lambda_{2}$ and $\lambda_{h}$ are of order unity. These masses can not be significantly smaller than $m_{\text {soft }}$ which is presumed to be of order the weak scale, and thus the non-negative integers $l, k, n$ are required to satisfy

$$
n \leq l, \quad k \leq l
$$

For the case with $n=l$, the Higgsino mass $\mu_{H}$ is of order $m_{\text {soft }}$, and then $H_{u}$ and $H_{d}$ can be interpreted as the MSSM Higgs doublets which have the weak scale VEVs generating the masses of $W, Z$, and also of the quarks and leptons:

$$
\left\langle H_{u}\right\rangle \approx\left\langle H_{d}\right\rangle \approx m_{\mathrm{soft}} \text { for } n=l .
$$

In this case, the observed quark mass spectrum gives

$$
\operatorname{det}\left(\lambda_{u} \lambda_{d}\right) \approx 10^{-16} \text { for } n=l .
$$

However for $l>n$, we have $\mu_{H} \gg m_{\text {soft }}$ and then $H_{u}$ and $H_{d}$ should be interpreted as additional massive Higgs doublets other than the MSSM Higgs doublets. Such additional (superheavy) Higgs doublets appear quite often in SUSY $E_{6}$ models and also string orbifold models [9]. At any rate, for $\mu_{H} \gg m_{\text {soft }}, H_{u}$ and $H_{d}$ have vanishing VEVs:

$$
\left\langle H_{u}\right\rangle=\left\langle H_{d}\right\rangle=0 \text { for } \quad l>n,
$$

and then $\lambda_{u}$ and $\lambda_{d}$ are not constrained at all. As a final remark on the vacuum configuration, one can explicitly confirm that

$$
\left\langle F_{i}\right\rangle=\left\langle\frac{\partial W}{\partial \phi_{i}}\right\rangle \approx m_{\mathrm{soft}}\left\langle\phi_{i}\right\rangle,
$$

for the models under consideration, which would assure the expression (9) for the superfield VEVs.

Given the above described vacuum configuration, the $N_{h}$ flavors of additional isosinglet quarks $\left(h+h^{c}\right)$ affect the QCD $\beta$ function at energy scales above $\mu_{h}$, particularly make it positive if $N_{h}>3$. Obviously we are then interested in small instantons with $\rho \lesssim \mu_{h}^{-1}$. To be definite, we will consider here only the three distinctive values of $N_{h}$ : the case with one $h+h^{c}$ in each generation $\left(N_{h}=3\right)$, the case with two $h+h^{c}$ in each generation $\left(N_{h}=6\right)$, and the case with three $h+h^{c}$ in each generation $\left(N_{h}=9\right)$. We then require that the QCD fine structure constant $\alpha$ does not blow up at energy scales below $M_{G U T}=2 \times 10^{16} \mathrm{GeV}$ for 
$N_{h}=6$ and $N_{h}=9$ which is a conservative condition. We do not require no blowing up of $\alpha$ above $M_{G U T}$ to $M_{P}$ because there is a possibility that enlarged gauge group at $M_{G U T}$ can change the running behavior of $\alpha$. This leads to the following conditions on $\mu_{h}$ and thus on $k$ and $l$ :

$$
\begin{aligned}
& N_{h}=6: \mu_{h} \gtrsim 10^{-5}\left(m_{\text {soft }} M_{G U T}\right)^{1 / 2} \rightarrow \frac{k+1}{l+1} \lesssim \frac{9}{10}, \\
& N_{h}=9: \mu_{h} \gtrsim 5 \times 10^{-4}\left(m_{\text {soft }} M_{G U T}^{2}\right)^{1 / 3} \rightarrow \frac{k+1}{l+1} \lesssim \frac{29}{45} .
\end{aligned}
$$

Within the range $l \leq 2$ which we will concentrate on, all possible sets of $(l, k)$ are restricted to $(1,0),(2,0),(2,1)$ for $N_{h}=6$ and $(1,0),(2,0)$ for $N_{h}=9$ by the above constraints.

As we will see, the axion potential induced by small instantons is highly sensitive to the Yukawa couplings $\lambda_{i}(i=u, d, 1,2, h)$. For $\lambda_{1}, \lambda_{2}$, and $\lambda_{h}$ which are unknown in any case, we simply assume that they are all of order unity. Note that this corresponds to a conservative choice when applied to check whether the resulting high energy axion potential satisfies the strong CP condition (5). About $\lambda_{u}$ and $\lambda_{d}$, we have two possibilities. For the case with $n=l, H_{u}$ and $H_{d}$ are the usual MSSM Higgs doublets and thus $\operatorname{det}\left(\lambda_{u} \lambda_{d}\right) \approx 10^{-16}$. However for $l>n, H_{u}$ and $H_{d}$ are additional (superheavy) Higgs doublets with vanishing VEVs, and then $\lambda_{u}$ and $\lambda_{d}$ can be assumed to be of order unity once again as a conservative choice.

Small instanton-induced axion potential has the tunnelling factor $e^{-\frac{2 \pi}{\alpha(\rho)}}$ for the single instanton contribution $\left(n_{1}=n_{2}=1\right)$ which gives the most dominant effect. This tunnelling factor can be determined by the one-loop $\beta$ function:

$$
\beta(E)=E \frac{d g}{d E}=-b_{0}(E) \frac{g^{3}}{16 \pi^{2}},
$$

where $b_{0}(E)=3$ for $E \lesssim \mu_{h}$, but $b_{0}(E)=3-N_{h}$ for $E \gtrsim \mu_{h}$. We then have

$$
\begin{aligned}
\exp (-2 \pi / \alpha(\rho)) & \approx\left(m_{\text {soft }} / \mu_{h}\right)^{3}\left(\rho \mu_{h}\right)^{3-N_{h}} \exp \left(-2 \pi / \alpha\left(m_{\text {soft }}\right)\right) \\
& \approx 2 \times 10^{-31}\left(\rho m_{\text {soft }}\right)^{3-N_{h}}\left(m_{\text {soft }} / M_{P}\right)^{\frac{N_{h}(l-k)}{l+1}},
\end{aligned}
$$

where we have used the expression (15) for $\mu_{h}$ together with the numerical value of $\exp \left(-2 \pi / \alpha\left(m_{\text {soft }}\right)\right) \approx 2 \times 10^{-31}$ for $m_{\text {soft }} \approx 1 \mathrm{TeV}$.

Another important ingredients in computing the instanton-induced axion potential are the selection rules dictated by the underlying theory at high energy scales around $\rho^{-1}$. ¿From the tree level superpotential, we can find various global symmetries under which both the superfields and the spurions transform. Low energy effective theory obtained by integrating out high momentum modes should preserve these symmetries. The global symmetries of the tree level superpotential (10) include

$$
\begin{aligned}
G= & S U(3)_{Q} \times S U(3)_{U^{c}} \times S U(3)_{D^{c}} \times S U\left(N_{h}\right)_{h} \times S U\left(N_{h}\right)_{h^{c}} \\
& \times U(1)_{A} \times U(1)_{X} \times U(1)_{X^{\prime}} \times U(1)_{R}
\end{aligned}
$$

where $S U(N)_{\Phi_{i}}$ denotes the $S U(N)$ rotation of the $N$-flavors of $\Phi_{i}$, and the $U(1)$-charges of all superfields and spurions are depicted in Table 1. 
Let us consider a $G$-invariant effective superpotential $e^{-Y} W_{\text {eff }}$ with a sensible limiting behavior, which would lead to an axion potential once the superfields are replaced by their VEVs. For a small instanton size $\rho \lesssim\langle S\rangle^{-1}$, it takes the following form:

$$
e^{-Y} W_{\text {eff }} \sim \rho^{-3} e^{-Y} \operatorname{det}\left(Z_{u} Z_{d} \tilde{Z}_{h}\right) \tilde{Z}_{1}^{N_{1}}\left(\rho^{2} H_{u} H_{d}\right)^{N_{2}}(\rho S)^{N_{3}} \tilde{Z}_{2}^{N_{4}},
$$

where

$$
\tilde{Z}_{h}=\frac{Z_{h}}{\left(\rho M_{P}\right)^{k}}, \quad \tilde{Z}_{1}=\frac{Z_{1}}{\left(\rho M_{P}\right)^{n}}, \quad \tilde{Z}_{2}=\frac{Z_{2}}{\left(\rho M_{P}\right)^{l}} .
$$

The integers $N_{i}(i=1,2,3,4)$ in the above are required to be non-negative to have a sensible limiting behavior. This condition can not be compatible with the $U(1)_{R^{-}}$selection rule

$$
N_{1}+N_{4}+2=0
$$

and thus no such an effective superpotential is allowed.

Since it does not have to be holomorphic, a generic instanton-induced Kähler potential which would lead to an axion potential can be written as

$$
e^{-Y} K_{\mathrm{eff}}=\rho^{-2} e^{-Y} \operatorname{det}\left(Z_{u} Z_{d} \tilde{Z}_{h}\right)\left(\begin{array}{c}
\tilde{Z}_{1} \\
\tilde{Z}_{1}^{*}
\end{array}\right)^{N_{1}}\left(\begin{array}{c}
\rho^{2} H_{u} H_{d} \\
\rho^{2} H_{u}^{*} H_{d}^{*}
\end{array}\right)^{N_{2}}\left(\begin{array}{c}
\rho S \\
\rho S^{*}
\end{array}\right)^{N_{3}}\left(\begin{array}{c}
\tilde{Z}_{2} \\
\tilde{Z}_{2}^{*}
\end{array}\right)^{N_{4}}\left(\begin{array}{c}
\rho D_{\alpha} D^{\alpha} \\
\rho \bar{D}_{\alpha} \bar{D}^{\alpha}
\end{array}\right)^{N_{5}} F(\eta)
$$

where $F(\eta)$ is a function of the real spurion $\eta=1+m_{0}^{2} \theta^{2} \bar{\theta}^{2}$ and the supercovariant derivatives are understood to be applied for either the superfields or the spurions. The $G$-selection rules require first of all the factor $\operatorname{det}\left(Z_{u} Z_{d} \tilde{Z}_{h}\right)$. One may then insert either $N_{1}$-powers of $Z_{1}$ or its complex conjugate, $N_{2}$-powers of $H_{u} H_{d}$ or its complex conjugate, and so on, to make $K_{\text {eff }}$ to be fully $G$-invariant. To make the notation simpler, in the following we will use the notation in which a negative power corresponds to the positive power of the complex conjugated fields or spurions. In this notation, the above Kähler potential can be written as

$$
e^{-Y} K_{\mathrm{eff}}=\rho^{-2} e^{-Y} \operatorname{det}\left(Z_{u} Z_{d} \tilde{Z}_{h}\right) \tilde{Z}_{1}^{N_{1}}\left(\rho^{2} H_{u} H_{d}\right)^{N_{2}}(\rho S)^{N_{3}} \tilde{Z}_{2}^{N_{4}}\left(\rho D^{2}\right)^{N_{5}} F(\eta),
$$

where now $\left\{N_{i}\right\}$ can be negative integers but still are constrained by the selection rules of $U(1)_{X}, U(1)_{X^{\prime}}$ and $U(1)_{R}$ as follows:

$$
\begin{aligned}
& N_{2}=N_{1}+3, \\
& N_{3}=(n+1) N_{1}+(l+3) N_{4}+(k+1) N_{h}, \\
& N_{5}=N_{1}+N_{4}+3 .
\end{aligned}
$$

In fact, there can be two additional suppression factors that are encoded in $F(\eta)$. The first one is the loop suppression factor which is always there, while the second one appears only when $\rho \lesssim f_{a}^{-1}$. Let $\phi_{P Q}$ denotes the scalar field responsible for the spontaneous $U(1)_{P Q^{-}}$ breaking. We then have

$$
\left\langle\phi_{P Q}\right\rangle=f_{a} e^{i a / f_{a} N_{D W}}
$$


where the positive integer $N_{D W}$ corresponds to the axion domain wall number. Then the $U(1)_{P Q}$-selection rule, i.e. the invariance under

$$
\begin{aligned}
\phi_{P Q} & \rightarrow e^{i \alpha} \phi_{P Q}, \\
\theta_{Q C D} & \rightarrow \theta_{Q C D}-\alpha N_{D W},
\end{aligned}
$$

requires that the axion potential induced by a very small instanton with $\rho \lesssim f_{a}^{-1}$ includes the factor $\left(\rho\left\langle\phi_{P Q}\right\rangle\right)^{N_{D W}}$. This factor is replaced by the simple phase factor $e^{i a / f_{a}}$ for larger instantons with $\rho \gtrsim f_{a}^{-1}$. Summarizing these, we have

$$
F(\eta=1) \approx \epsilon_{a}\left(\frac{1}{4 \pi^{2}}\right)^{L}
$$

where

$$
\epsilon_{a} \approx\left\{\begin{array}{ll}
\left(\rho f_{a}\right)^{N_{D W}} & \text { for } \rho \lesssim f_{a}^{-1}, \\
1 & \text { for } \rho \gtrsim f_{a}^{-1}
\end{array},\right.
$$

and $L$ denotes the number of loops in the instanton graph.

After replacing the superfields in $K_{\text {eff }}$ by their VEVs and also integrating over the Grassmann variable, we find

$$
\begin{aligned}
V_{\mathrm{HE}}(\rho) & \approx \rho^{-4} F(1) e^{-\frac{2 \pi}{\alpha(\rho)}}\left(\rho m_{\mathrm{soft}}\right)^{2+\left|N_{5}\right|}\left(\rho M_{P}\right)^{-N_{0}}\left(\rho^{2}\left\langle H_{u} H_{d}\right\rangle\right)^{\left|N_{2}\right|} \\
& \times(\rho\langle S\rangle)^{\left|N_{3}\right|} \operatorname{det}\left(\lambda_{u} \lambda_{d} \lambda_{h}\right) \lambda_{1}^{\left|N_{1}\right|} \lambda_{2}^{\left|N_{4}\right|}
\end{aligned}
$$

where

$$
N_{0}=k N_{h}+n\left|N_{1}\right|+l\left|N_{4}\right|
$$

Note that the above axion potential includes the model-independent SUSY suppression factor $\left(\rho m_{\text {soft }}\right)^{2}$ in addition to the tunnelling factor $e^{-\frac{2 \pi}{\alpha(\rho)}}$ and also the model-dependent suppression factors due to small field VEVs and couplings. Using the field VEVs (14), (17) and (19) together with (23) and (33), we finally arrive at

$$
\begin{aligned}
V_{\mathrm{HE}}(\rho) & \approx 2 \times 10^{-31} \epsilon_{a} \rho^{-4}\left(\rho m_{\mathrm{soft}}\right)^{5+N_{S}-N_{h}}\left(\rho M_{P}\right)^{-N_{0}+\frac{l}{l+1}\left|N_{3}\right|} \\
& \times\left(\frac{1}{4 \pi^{2}}\right)^{L}\left(\frac{m_{\mathrm{soft}}}{M_{P}}\right)^{\frac{N_{h}(l-k)}{l+1}} \operatorname{det}\left(\lambda_{u} \lambda_{d} \lambda_{h}\right) \lambda_{1}^{\left|N_{1}\right|} \lambda_{2}^{\left|N_{4}\right|}
\end{aligned}
$$

where

$$
N_{S}= \begin{cases}2\left|N_{2}\right|+\frac{1}{l+1}\left|N_{3}\right|+\left|N_{5}\right| & \text { for } l=n \\ \frac{1}{l+1}\left|N_{3}\right|+\left|N_{5}\right| & \text { for } l>n\end{cases}
$$

Here the loop number $L$ can be determined by looking at an explicit instanton graph yielding the above form of axion potential. Note that $\left\langle H_{u}\right\rangle \approx\left\langle H_{d}\right\rangle \approx m_{\text {soft }}$ only for $l=n$. For other cases with $l>n$, we have $\left\langle H_{u}\right\rangle=\left\langle H_{d}\right\rangle=0$ and thus only $N_{2}=0$ can yields a nonvanishing axion potential. 
Given the values of $N_{h}=3,6,9$ and $(n, l, k)$ satisfying the constraints (16) and (21), we can now pick out the values of $\left\{N_{i}\right\}(i=1 \sim 5)$ which would give the most dominant contribution to $V_{\mathrm{HE}}$ for a fixed value of $\rho$. It turns out that for all cases studied here such values of $\left\{N_{i}\right\}$ lead to a negative total power of $\rho$ in $V_{\mathrm{HE}}$ and thus smaller instantons give a larger contribution. Since we wish to see whether $V_{\mathrm{HE}}$ is small enough to satisfy the strong CP condition (5), we are interested in the largest possible value of $V_{\mathrm{HE}}$, i.e. an upper bound. We thus take $\rho \approx 1 / M_{P}$, being the smallest possible value of $\rho$, and also did not take into account the $f_{a}$-dependent suppression factor $\epsilon_{a} \approx\left(\rho f_{a}\right)^{N_{D W}}$ in our numerical study. It is then clear from eq. (37) that, for the fixed values of $\left(N_{h}, n, l, k\right)$ and $\rho M_{P} \approx 1$, the largest possible value of $V_{\mathrm{HE}}$ is obtained for the minimal values of $N_{S}$ and also of the loop number $L$. In each case, it is rather straightforward to find the minimal value of $N_{S}$ and also the corresponding instanton graph with a minimal number of loops. (See Figures 2, 4, 5, 6.) The results are summarized in Table 2 for all cases with $l \leq 2$. It turns out that $N_{S}<2$ in all cases with $l=n \leq 2$ and thus $N_{2}=0$ even when $l=n$. We stress that the values of $V_{\mathrm{HE}}$ in Table 2 are obtained for the smallest possible value of $\rho\left(\approx M_{P}^{-1}\right)$ and the conservative choice of the loop factor $\left(\approx \frac{1}{4 \pi^{2}}\right)$, and also the possible additional suppression factor $\epsilon_{a} \approx\left(\rho f_{a}\right)^{N_{D W}}$ for $f_{a} \lesssim \rho^{-1}$ was not taken into account. In this sense, they could be largely overestimated and thus should be understood as a kind of upperbound on $V_{\mathrm{HE}}$.

As we have pointed out, a naive instanton graph analysis can yield a misleading result with less power of $\rho m_{\text {soft }}$ than what is required by SUSY and the internal symmetry selection rules. Such a graph should be cancelled by other instanton graphs which are related to each other by SUSY. Avoiding this complication was the main motivation to introduce a manifestly supersymmetric scheme to compute the small instanton-induced axion potential. To make the motivation more clear, let us consider some examples of misleading instanton amplitudes. For the case of $(n, l, k)=(0,1,0)$ and $N_{h}=9$, there can be a diagram like Fig. 1 which is consistent with the selection rules of all internal symmetries, but includes only one insertion of $\rho m_{\text {soft }}$ (the $A$ parameter in this case). The size of the resulting amplitude is estimated to be (for $\rho^{-1} \approx f_{a} \approx M_{P}$ )

$$
\begin{aligned}
V_{\mathrm{HE}}^{\mathrm{mis}} & \approx\left(\frac{1}{4 \pi^{2}}\right)^{12} e^{-\frac{2 \pi}{\alpha\left(M_{P}\right)}} M_{P} \operatorname{det}\left(\lambda_{u} \lambda_{d} \lambda_{h}\right) \lambda_{1}^{* 3} \lambda_{2}^{*}\langle S\rangle^{2} A \\
& \approx 6 \times 10^{15} \operatorname{det}\left(\lambda_{u} \lambda_{d} \lambda_{h}\right) \lambda_{1}^{* 3} \lambda_{2}^{*}(\mathrm{GeV})^{4},
\end{aligned}
$$

which would be much larger than $10^{-9} f_{\pi}^{2} m_{\pi}^{2}$ if all the Yukawa couplings are taken to be of order unity. (Note that $l>n$ in this case and thus $H_{u}$ and $H_{d}$ should be interpreted as the superheavy Higgs doublets which have a vanishing VEV and thus can have a large Yukawa coupling with the standard model quarks.) Based on this result, one may conclude that the axion solution to the strong $\mathrm{CP}$ problem can be spoiled by small size QCD instantons in this model. However to be consistent with both the SUSY and the internal symmetry selection rules, the instanton amplitude should include at least two powers of $\rho m_{\text {soft }}$ and thus the above amplitude should be cancelled by other instanton amplitudes. Our manifestly supersymmetric scheme tells us that the most dominant contribution comes from Fig. 2 including three insertions of $\rho m_{\text {soft }}$. The resulting correct $V_{\mathrm{HE}}$ is then estimated to be

$$
\begin{aligned}
V_{\mathrm{HE}} & \approx\left(\frac{1}{4 \pi^{2}}\right)^{11} e^{-\frac{2 \pi}{\alpha\left(M_{P}\right)}} \frac{m_{\mathrm{soft}}^{2}}{M_{P}} \operatorname{det}\left(\lambda_{u} \lambda_{d} \lambda_{h}\right) \lambda_{1}^{* 3} \lambda_{2}^{*}\langle S\rangle^{2} A \\
& \approx 6 \times 10^{-14} \operatorname{det}\left(\lambda_{u} \lambda_{d} \lambda_{h}\right) \lambda_{1}^{* 3} \lambda_{2}^{*}(\mathrm{GeV})^{4},
\end{aligned}
$$


which is smaller than $10^{-9} f_{\pi}^{2} m_{\pi}^{2}$ even when all Yukawa couplings are taken to be of order unity. In fact, in some cases one can draw an instanton graph without any insertion of $\rho m_{\text {soft }}$. For instance, for the case with $N_{h}=3$ and $n=l=k=0$, Fig. 3 provides an instanton amplitude which is consistent with all the internal symmetry selection rules but does not contain any $\rho m_{\text {soft }}$. Once again, such amplitude should be cancelled and in the manifestly supersymmetric scheme the dominant contribution is from Fig. 4 including two insertions of $\rho m_{\text {soft }}$.

Let us finally comment a technical point about the number of loops in the instanton graph. When two graphs have the same value of $N_{S}$, the dominant contribution comes from the one with a smaller number of loops. For instance, for the case with $N_{h}=9$ and $(n, l, k)=(1,1,0)$, both $\left(N_{1}, N_{3}, N_{4}, N_{5}\right)=(-3,3,0,0)$ and $(-3,-1,-1,-1)$ give the same value of $N_{S}=3 / 2$. Fig. 5 shows the diagram of $(-3,3,0,0)$ with the number of loops $L=11$, while Fig. 6 is for $(-3,-1,-1,-1)$ with the number of loops $L=13$. In this example, Fig. 5 has two more scalar VEVs $(\langle S\rangle)$ than Fig. 6, but has two smaller number of loops. Usually a graph with more scalar VEVs has a fewer scalar loops because the number of scalar loops can be reduced by closing the scalar field line by inserting its VEV. Thus when there are two or more graphs with the same $N_{S}$, the graph with a more scalar VEVs has a fewer loops and thus gives a dominant contribution.

To close all the quark zero modes in the instanton graph, one needs the insertion of either the bare quark mass or the Yukawa coupling involving a generic complex scalar field $\phi$. In our case, there is no bare quark mass and thus all the quark zero modes are closed by the Yukawa couplings. As a result, the instanton graph involves many $\phi$-lines which should be closed again to yield a vacuum amplitude. There are many ways to close the $\phi$-lines. One may close one $\phi$-line without yielding any scalar loop by the insertion of $\langle\phi\rangle$, or close two $\phi$-lines by inserting a complex mass of the form $M_{\phi}^{2} \phi^{2}+$ h.c. which would yield one scalar loop. Inserting the couplings involving higher powers of $\phi$ to close the $\phi$-lines results in more scalar loops. Obvioulsy the insertion of $\langle\phi\rangle$ in the instanton graph leads to the suppression by $\rho\langle\phi\rangle$. Also in supersymmetrc models, generically $M_{\phi}^{2} \approx m_{\text {soft }} M_{\psi}$ where $M_{\psi}$ is a supersymmetric mass of the fermionic partner $\psi$ of $\phi$, and thus the insertion of $M_{\phi}^{2}$ leads to a suppression by $\rho m_{\text {soft }}$. Because of these suppressions, although economical in reducing the scalar loops, instanton graphs in which most of the scalar lines are closed either by $\langle\phi\rangle$ or $M_{\phi}^{2}$ do not give a dominant contribution to $V_{\mathrm{HE}}$. This is the reason why in our cases the dominant contribution comes from the instanton graph involving relatively many scalar loops.

\section{CONCLUSION}

In this paper, we have studied the high energy axion potential $V_{\mathrm{HE}}$ induced by small QCD instantons in supersymmetric models in which QCD is not asymtotically free at high energy scales. In such models, $V_{\mathrm{HE}}$ may be larger than $10^{-9} f_{\pi}^{2} m_{\pi}^{2}$ and then the axion solution to the strong CP problem can not be successfully implemented. To avoid the difficulty arising from the possible SUSY cancellation between different instanton graphs, we introduced a manifestly supersymmetric scheme to compute $V_{\mathrm{HE}}$ in generic SUSY models and applied it for a class of illustrative models. In our scheme, small instanton effects can be summarized by the effective superpotential $\left(W_{\text {eff }}\right)$ and also by the effective Kähler potential $\left(K_{\text {eff }}\right)$. By 
imposing the selection rules and a sensible limiting behavior together with the holomorphy, we showed that $W_{\text {eff }}$ does not allow a term which would contribute to the axion potential and thus $V_{\mathrm{HE}}$ always comes from $K_{\mathrm{eff}}$. As a simple consequence, $V_{\mathrm{HE}}$ is suppressed at least by two powers of $\rho m_{\text {soft }}$ where $\rho$ denotes the small instanton size and $m_{\text {soft }}$ is the soft SUSY breaking mass which is presumed to be of order the weak scale. In addition to this model-independent suppression, $V_{\mathrm{HE}}$ can be suppressed further by (i) small field VEVs $\left\langle\rho \phi_{i}\right\rangle$, (ii) small Yukawa couplings, and also (iii) loop factors. These model-dependent suppression factors are carefully analyzed for a class of illustrative models to estimate the possible largest value of $V_{\mathrm{HE}}$ and the results are summarized in Table 2. Our analysis suggests that the axion solution to the strong CP problem is stable against the effects of small instantons in a wide class of SUSY models even when QCD is not asymtotically free at high energy scales.

\section{ACKNOWLEDGMENTS}

HK would like to thank C. Munoz for helpful discussions. This work is supported in part by KOSEF Grant 981-0201-004-2, MOE Basic Science Institute Program BSRI-982434, KOSEF through CTP of Seoul National University, and KRF under the Distinguished Scholar Exchange Program. 


\section{REFERENCES}

[1] J. E. Kim, Phys. Rep. 150 (1987) 1; H.-Y. Cheng, Phys. Rep. 158 (1988) 1; R. D. Peccei, in CP Violation, ed. C. Jarlskog (World Scientific, Singapore, 1989); R. D. Peccei, hep-ph/9807516.

[2] R. D. Peccei and H. R. Quinn, Phys. Rev. Lett. 38 (1977) 1440; Phys. Rev. D 16 (1977) 1791

[3] M. Dine and N. Seiberg, Nucl. Phys. B 273 (1986) 109.

[4] J. Flynn and L. Randall, Nucl. Phys. B 293 (1987) 731.

[5] H. Georgi and L. Randall, Nucl. Phys. B 276 (1986) 241.

[6] H. P. Nilles Phys. Rep. 110 (1984) 1.

[7] M. Dine and A. E. Nelson, Phys. Rev. D 48 (1993) 1277; M. Dine, A. E. Nelson and Y. Shirman, Phys. Rev. D 51 (1995) 1362. M. Dine, A. E. Nelson, Y. Nir and Y. Shirman, Phys. Rev. D 53 (1996) 2658.

[8] K. Choi, H. B. Kim, and J. E. Kim, Nucl. Phys. B 490 (1997) 349.

[9] C. Munoz, private communications. 


\section{TABLES}

TABLE I. Quantum numbers of superfields and spurions

\begin{tabular}{|c||cccc|}
\hline \hline & $U(1)_{A}$ & $U(1)_{X}$ & $U(1)_{X^{\prime}}$ & $U(1)_{R}$ \\
\hline$Q$ & 1 & 0 & 0 & 1 \\
\hline$u^{c}, d^{c}$ & 1 & -1 & 0 & 1 \\
\hline$h, h^{c}$ & 1 & 0 & 0 & 1 \\
\hline$H_{u}, H_{d}$ & 0 & 1 & 0 & 0 \\
\hline$S$ & 0 & 0 & 1 & 6 \\
\hline$e^{-Y}$ & $12+2 N_{h}$ & -6 & 0 & 0 \\
\hline$Z_{u}, Z_{d}$ & -2 & 0 & 0 & 0 \\
\hline$Z_{h}$ & -2 & 0 & $-(k+1)$ & 2 \\
\hline$Z_{1}$ & 0 & -2 & $-(n+1)$ & 2 \\
\hline$Z_{2}$ & 0 & 0 & 0 & -2 \\
\hline$d^{2} \theta$ & 0 & 0 & & \\
\hline \hline
\end{tabular}


TABLE II. The largest possible size of $V_{\mathrm{HE}}$ and $\delta=V_{\mathrm{HE}} / 10^{-9} f_{\pi}^{2} m_{\pi}^{2}$ in each model. If $\delta \lesssim 1$, the axion solution to the strong CP problem is untouched by small instantons.

\begin{tabular}{|c|c|c|c|c|c|c|c|c|}
\hline$N_{h}$ & $n$ & $l$ & $k$ & $\left(N_{1}, N_{3}, N_{4}, N_{5}\right)$ & $N_{S}$ & $L($ loop $)$ & $V_{\mathrm{HE}}(\mathrm{GeV})^{4}$ & $\delta$ \\
\hline 3 & 0 & 0 & 0 & $(-3,0,0,0)$ & 0 & 8 & $1.6 \times 10^{-17}$ & $4.1 \times 10^{-5}$ \\
\hline 3 & 0 & 1 & 0 & $(-3,0,0,0)$ & 0 & 8 & $1.8 \times 10^{-24}$ & $4.5 \times 10^{-12}$ \\
\hline 3 & 0 & 1 & 1 & $(-3,3,0,0)$ & $3 / 2$ & 8 & $1.8 \times 10^{-24}$ & $4.5 \times 10^{-12}$ \\
\hline 3 & 0 & 2 & 0 & $(-3,0,0,0)$ & 0 & 8 & $4.1 \times 10^{-32}$ & $1.0 \times 10^{-19}$ \\
\hline 3 & 0 & 2 & 1 & $(-3,3,0,0)$ & 1 & 8 & $4.1 \times 10^{-32}$ & $1.0 \times 10^{-19}$ \\
\hline 3 & 0 & 2 & 2 & $(-3,1,-1,-1)$ & $4 / 3$ & 12 & $2.7 \times 10^{-28}$ & $6.6 \times 10^{-16}$ \\
\hline 3 & 1 & 1 & 0 & $(-3,-3,0,0)$ & $3 / 2$ & 8 & $2.0 \times 10^{-63}$ & $5.1 \times 10^{-51}$ \\
\hline 3 & 1 & 1 & 1 & $(-3,0,0,0)$ & 0 & 11 & $2.6 \times 10^{-22}$ & $6.6 \times 10^{-10}$ \\
\hline 3 & 1 & 2 & 0 & $(-3,-3,0,0)$ & 1 & 8 & $2.0 \times 10^{-47}$ & $5.1 \times 10^{-35}$ \\
\hline 3 & 1 & 2 & 1 & $(-3,0,0,0)$ & 0 & 11 & $1.3 \times 10^{-21}$ & $3.3 \times 10^{-9}$ \\
\hline 3 & 1 & 2 & 2 & $(-3,3,0,0)$ & 1 & 11 & $1.3 \times 10^{-21}$ & $3.3 \times 10^{-9}$ \\
\hline 3 & 2 & 2 & 0 & $(-3,-1,1,1)$ & $4 / 3$ & 12 & $6.6 \times 10^{-75}$ & $1.7 \times 10^{-62}$ \\
\hline 3 & 2 & 2 & 1 & $(-3,-3,0,0)$ & 1 & 11 & $6.6 \times 10^{-53}$ & $1.7 \times 10^{-40}$ \\
\hline 3 & 2 & 2 & 2 & $(-3,0,0,0)$ & 0 & 14 & $4.3 \times 10^{-27}$ & $1.1 \times 10^{-14}$ \\
\hline 6 & 0 & 1 & 0 & $(-3,3,0,0)$ & $3 / 2$ & 8 & $1.8 \times 10^{-24}$ & $4.5 \times 10^{-12}$ \\
\hline 6 & 0 & 2 & 0 & $(-3,3,0,0)$ & 1 & 8 & $4.1 \times 10^{-32}$ & $1.0 \times 10^{-19}$ \\
\hline 6 & 0 & 2 & 1 & $(-3,4,-1,-1)$ & $7 / 3$ & 12 & $2.7 \times 10^{-28}$ & $6.6 \times 10^{-16}$ \\
\hline 6 & 1 & 1 & 0 & $(-3,0,0,0)$ & 0 & 11 & $2.6 \times 10^{-22}$ & $6.6 \times 10^{-10}$ \\
\hline 6 & 1 & 2 & 0 & $(-3,0,0,0)$ & 0 & 11 & $1.3 \times 10^{-21}$ & $3.3 \times 10^{-9}$ \\
\hline 6 & 1 & 2 & 1 & $(-3,1,-1,-1)$ & $4 / 3$ & 15 & $8.6 \times 10^{-18}$ & $2.2 \times 10^{-5}$ \\
\hline 6 & 2 & 2 & 0 & $(-3,-3,0,0)$ & 1 & 11 & $6.6 \times 10^{-53}$ & $1.7 \times 10^{-40}$ \\
\hline 6 & 2 & 2 & 1 & $(-3,-3,0,0)$ & 1 & 14 & $4.3 \times 10^{-27}$ & $1.1 \times 10^{-14}$ \\
\hline 9 & 0 & 1 & 0 & $(-3,2,-1,-1)$ & 2 & 11 & $5.9 \times 10^{-14}$ & $1.5 \times 10^{-1}$ \\
\hline 9 & 0 & 2 & 0 & $(-3,1,-1,-1)$ & $4 / 3$ & 12 & $2.7 \times 10^{-28}$ & $6.6 \times 10^{-16}$ \\
\hline 9 & 1 & 1 & 0 & $(-3,3,0,0)$ & $3 / 2$ & 11 & $2.6 \times 10^{-22}$ & $6.6 \times 10^{-10}$ \\
\hline 9 & 1 & 2 & 0 & $(-3,3,0,0)$ & 1 & 11 & $1.3 \times 10^{-21}$ & $3.3 \times 10^{-9}$ \\
\hline 9 & 2 & 2 & 0 & $(-3,0,0,0)$ & 0 & 14 & $4.3 \times 10^{-27}$ & $1.1 \times 10^{-14}$ \\
\hline
\end{tabular}




\section{FIGURES}

FIG. 1. Instanton graph which is consistent with all the internal symmetry selection rules but is required to be cancelled by SUSY for the case of $N_{h}=9$ and $(n, l, k)=(0,1,0)$. Here the solid lines with and without waves denote the gluino and quark modes, respectively, while the dotted lines are the scalar fluctuations of Higgs, squarks, and $S$. The dark blobs represent the insertions of complex couplings or field VEVs which are explicitly written in the graph.

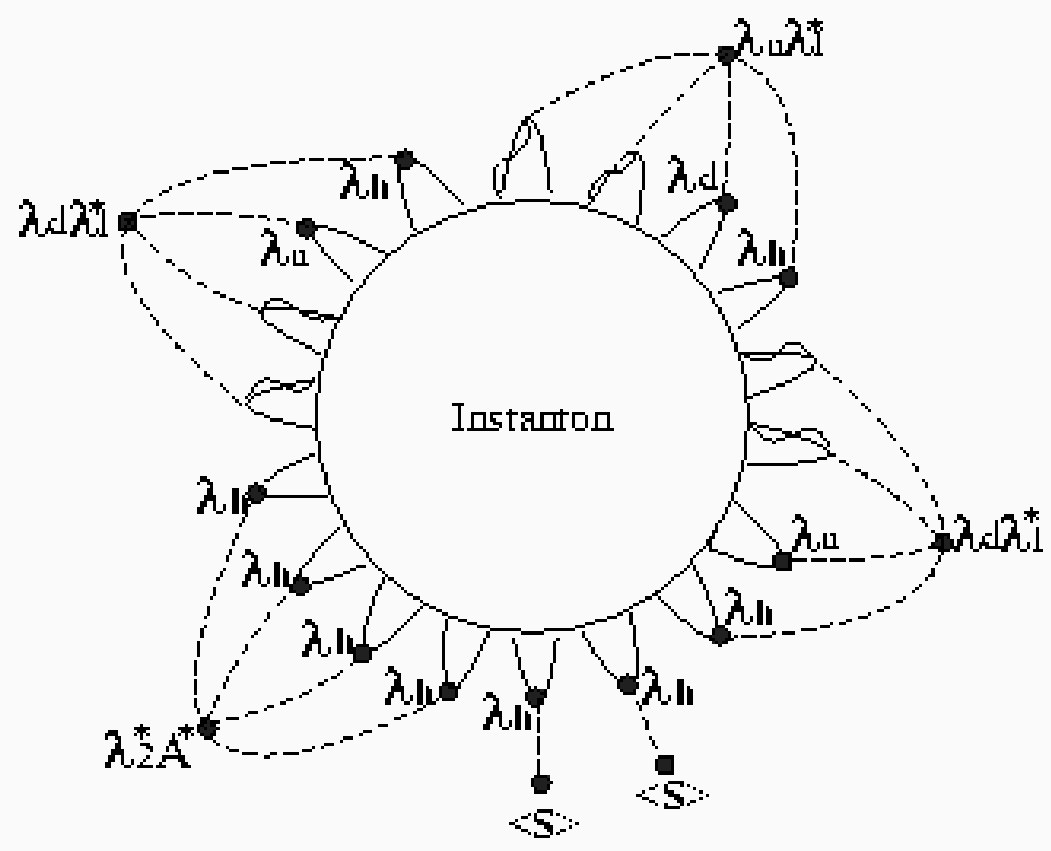

FIG. 2. Dominant instanton graph for the case of $N_{h}=9$ and $(n, l, k)=(0,1,0)$.

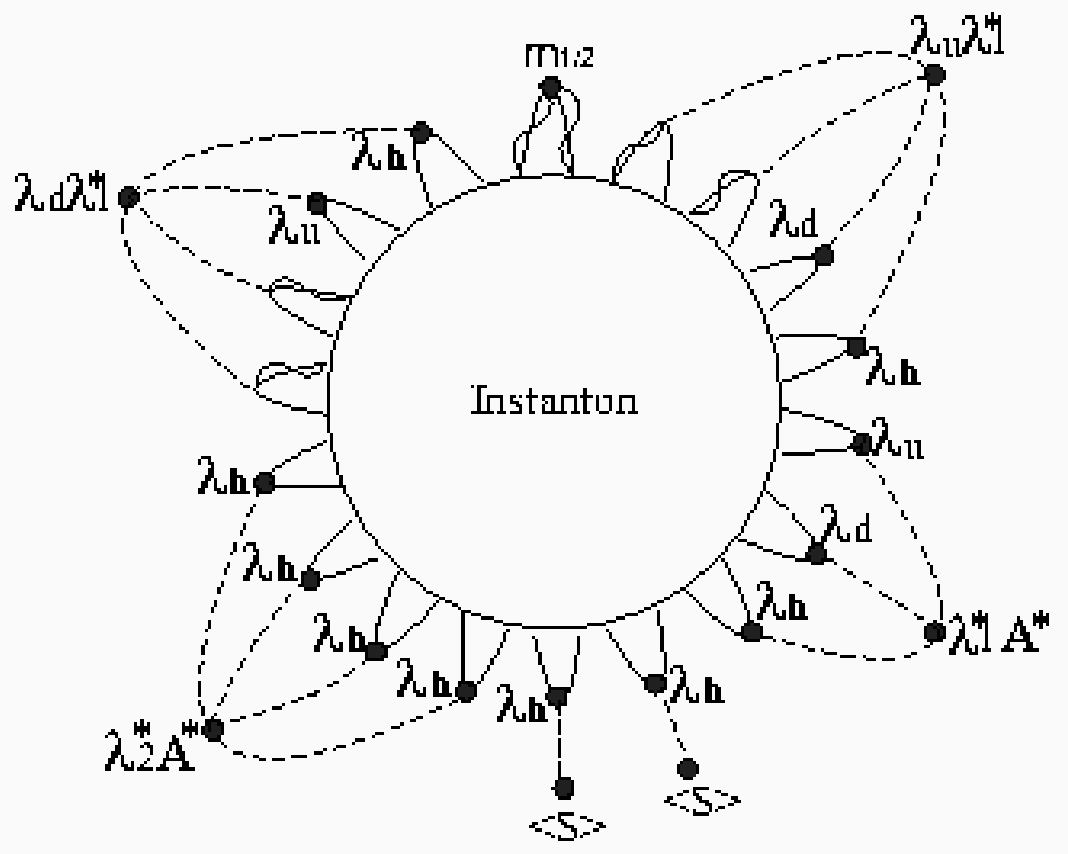


FIG. 3. Instanton graph which is required to be cancelled by SUSY for the case of $N_{h}=3$ and $(n, l, k)=(0,0,0)$.

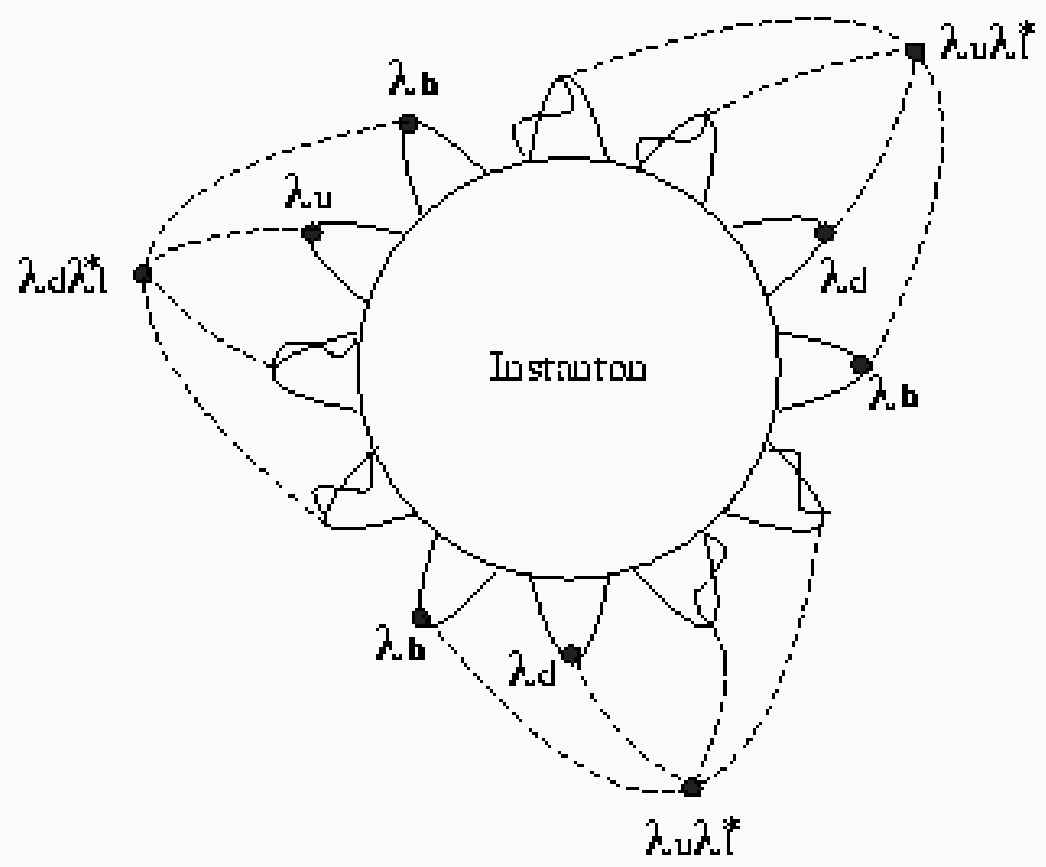

FIG. 4. Dominant instaton graph for the case of $N_{h}=3$ and $(n, l, k)=(0,0,0)$.

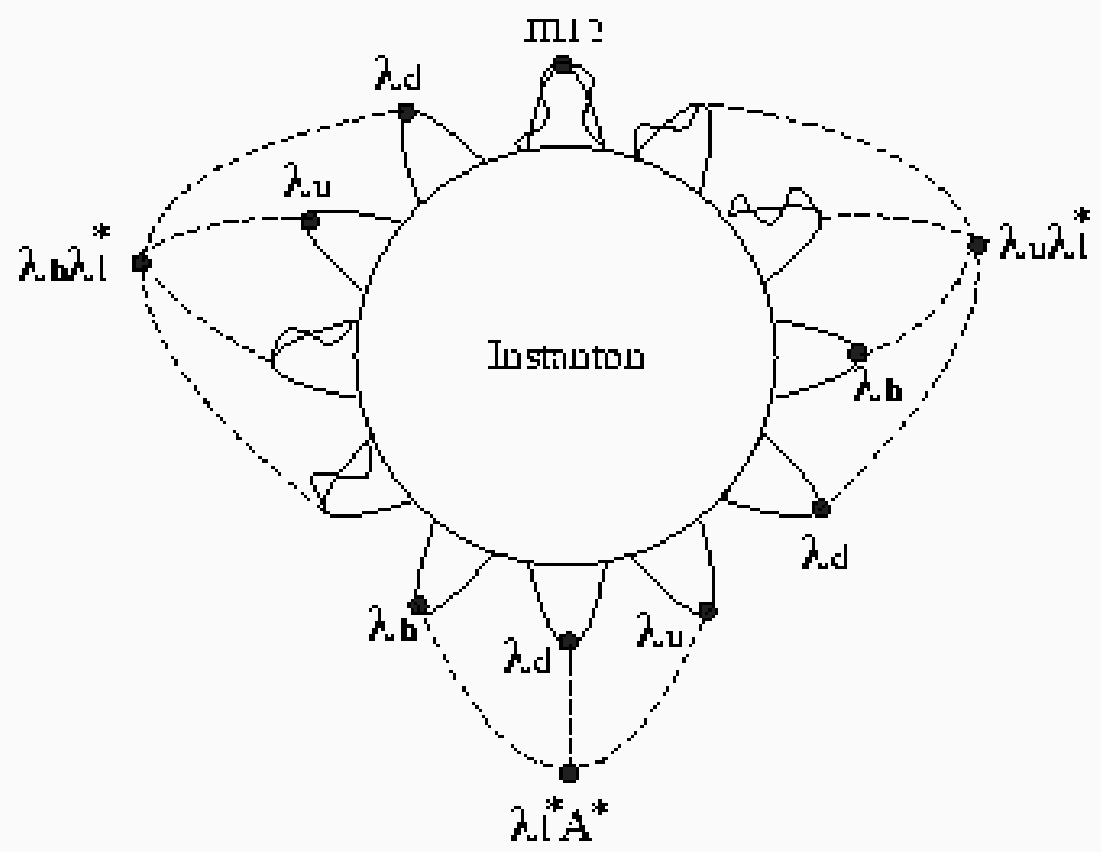


FIG. 5. Instanton graph with the minimal values of $N_{S}$ and the number of loops $L$ for the case of $N_{h}=9$ and $(n, l, k)=(1,1,0): N_{S}=3 / 2$ and $L=11$.

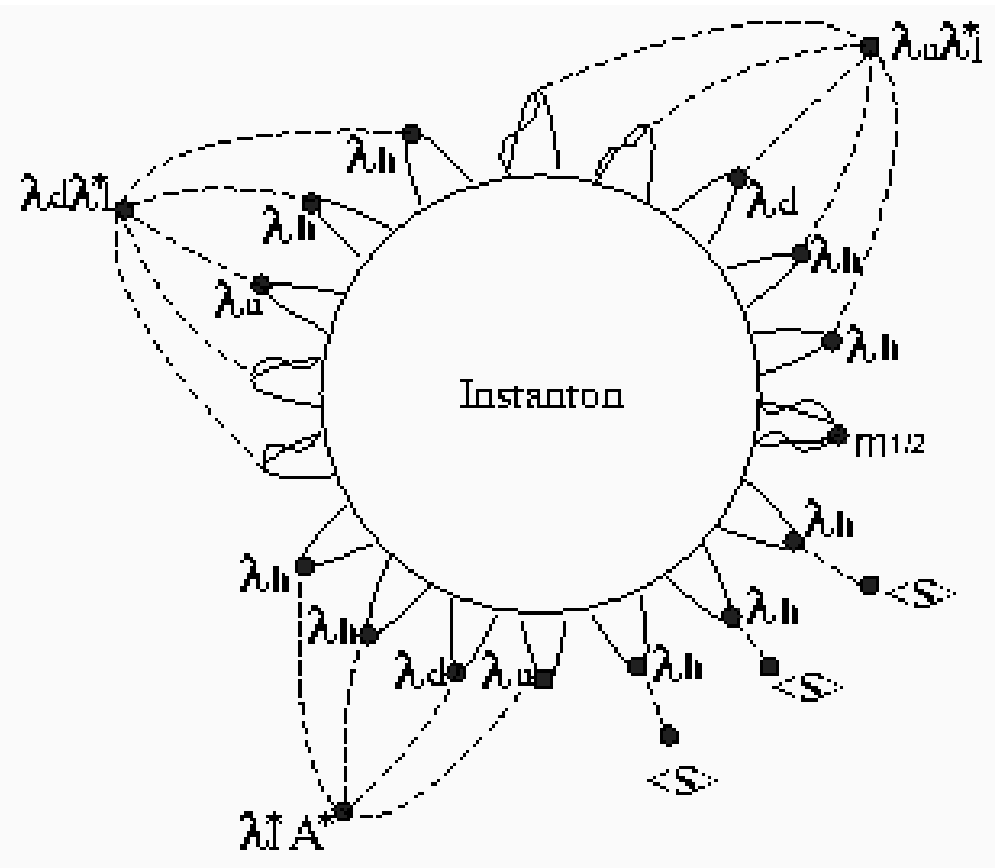

FIG. 6. Instanton graph with the same $N_{S}=3 / 2$ as Fig. 5 but a larger number of loops $L=13$ for the case of $N_{h}=9$ and $(n, l, k)=(1,1,0)$.

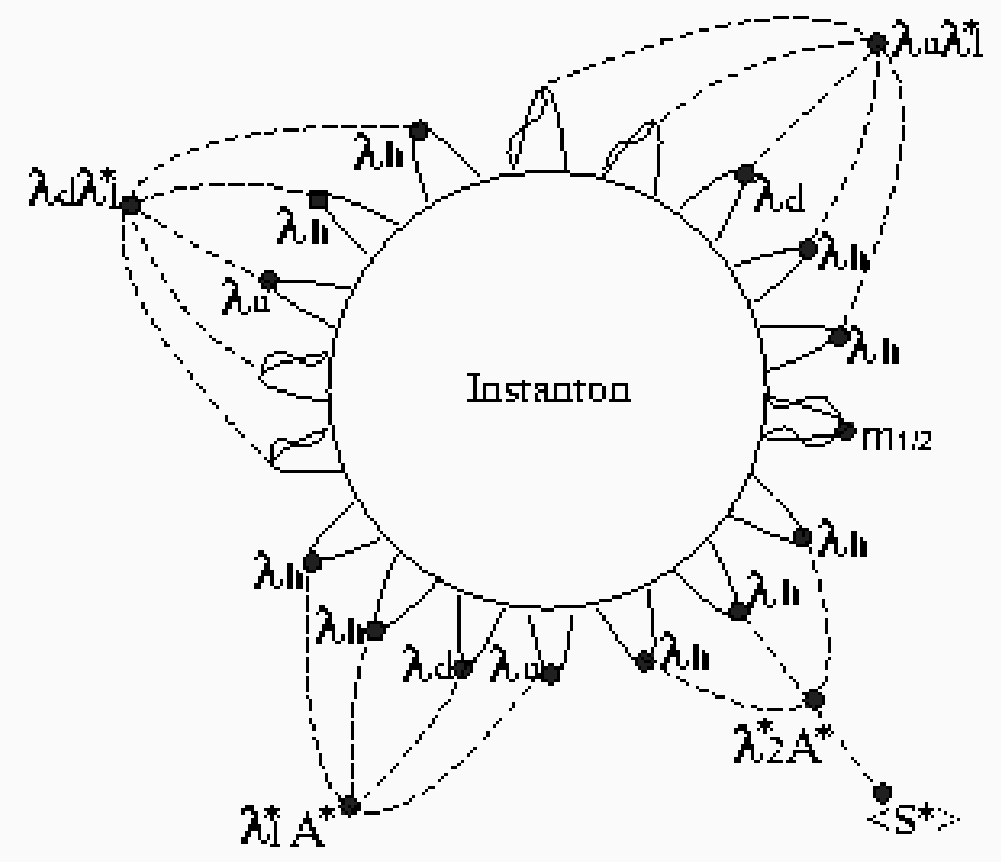

